This is a preprint of a paper published as:

Van Steen, C., Pahlavan, L., Wevers, M., Verstrynge, E. (2019). Localisation and characterisation of corrosion damage in reinforced concrete by means of acoustic emission and X-ray computed tomography. Construction and Building Materials, 197, 21-29.

Original DOI: 10.1016/j.conbuildmat.2018.11.159 


\title{
Localisation and characterisation of corrosion damage in reinforced concrete by means of acoustic emission and X-ray computed tomography
}

\author{
Charlotte Van Steen ${ }^{\mathrm{a}, *}$, Lotfollah Pahlavan ${ }^{\mathrm{b}},{\text { Martine } \text { Wevers }^{\mathrm{c}} \text {, Els Verstrynge }}^{\mathrm{a}}$ \\ ${ }^{a}$ Department of Civil Engineering, KU Leuven, Leuven, Belgium \\ ${ }^{b}$ Department of Maritime and Transport Technology, TU Delft, Delft, The Netherlands \\ ${ }^{c}$ Department of Metallurgy and Materials Engineering, $K U$ Leuven, Leuven, Belgium
}

\begin{abstract}
Many existing reinforced concrete (RC) structures are suffering from corrosion damage. The development of reliable tools to characterise and localise such damage is essential to assess the structural capacity. The acoustic emission (AE) technique has proven to be promising for this purpose. However, this technique poses challenges to reliably interpret the results and lacks rigourous validation. In this paper, microfocus X-ray computed tomography (micro-CT) is used to validate the use of $\mathrm{AE}$ for the localisation and characterisation of chlorideinduced corrosion damage. An experimental protocol is designed, where both techniques are applied on small-scale reinforced mortar samples during accelerated corrosion. In addition to the frequently applied localisation of the $\mathrm{AE}$ events, a new signal-based clustering algorithm of the AE events is proposed, which allows to distinguish between different damage processes, such as friction due to the emergence of corrosion products and corrosion-induced mortar cracking. The results obtained from the AE localisation and clustering are confirmed by the micro-CT images and are found to be in line with alternative damage characterisation techniques based on $\mathrm{AE}$ parameters, that are found in the literature. Finally, it is shown that the combination of clustered AE events and micro-CT for the considered small-scale samples can help to understand
\end{abstract}

\footnotetext{
${ }^{*}$ Corresponding author. Tel.: +32 16374521

Email address: charlotte.vansteen@kuleuven.be (Charlotte Van Steen)
} 
the different damage processes induced by corrosion.

Keywords: reinforcement corrosion, x-ray computed tomography, acoustic emission, clustering, localisation

\section{Introduction}

In many cases, limited maintenance is executed during service life of reinforced concrete (RC) structures. Whenever existing buildings and infrastructure are re-used for new functions (adaptive re-use), subjected to unforeseen dam5 age or deterioration, or their lifetime is extended, their structural safety and serviceability becomes a concern. Reinforcement corrosion is believed to be the most widespread deterioration mechanism in RC structures. As maintenance and repair costs are running high, development of reliable assessment tools becomes necessary. Normally the reinforcement is protected by the high alkaline environment of the surrounding concrete, which prevents the steel from corroding. However, this protective layer can be destroyed due to carbonation and/or chloride-ingress. During the corrosion process, the steel material dissolves leading to a reduced cross section of the reinforcement. Lower density corrosion products are formed at the same time causing internal tensile stresses in the concrete and eventually cracking and spalling of the concrete cover.

To allow the development of efficient performance-based approaches for existing structures, a shift towards quantifying the damage level and residual capacity is needed. On-site quantification of deterioration requires advanced nondestructive techniques (NDT) for material and damage characterisation and localisation. Electrochemical techniques such as open circuit potential monitoring (OPC), polarisation resistance, galvanostatic pulse method (GPM), and resistivity method, are widely used to monitor corrosion on-site. Unfortunately they are dependent on climatic conditions and might lack to provide precise information 1] 2]. This increases the demand for calibration with other tech25 niques.

A very promising technique to capture not only the corrosion process itself, 
but also the progress of concrete cracking is the acoustic emission (AE) technique [3]. Local stress redistributions in the material emit high frequency elastic waves that can be captured by piezoelectric sensors on the surface. Linking the arrival time of the wave at multiple sensors allows to localise the source. The technique has proven its efficiency for localising damage in metal structures such as pressure vessels and pipelines, where direct contact with the metal is possible 44 5]. AE monitoring has been successfully applied during rebar corrosion in concrete and destructive testing of corroded RC samples and components 6] 7] 8]. Still, a lot of assumptions of AE trends are very common in the field of structural materials and the technique lacks rigourous validation. To adress this, a second method is needed for validation of AE. A link is made in this paper with an advanced visualisation technique that can be applied on small samples, namely microfocus X-ray computed tomography (micro-CT) 9. Micro-CT is a non-destructive observation technique which allows to visualise the inner structure of a non-transparent specimen based on the attenuation of X-rays. In civil engineering, it is used to study the internal structure of porous building materials such as mortar, concrete paste, bricks and sandstone 10]11. For research on corrosion-induced cracking, X-ray measurements were applied by Michel et

45 al. [12 to visualise the development of the corrosion accommodating region (CAR). Savija et al. [13] applied micro-CT to monitor rust formation and cover cracking in addition to results of other techniques such as nanoindentation. Previous studies suggest that micro-CT is promising to obtain a visual confimation of AE data [14] [15] [16].

The aim of the study is to better understand AE results obtained during the corrosion process of reinforced concrete samples by combining localisation and clustering of AE events, and visual data from X-ray scans. First, a testing protocol is designed and validated that combines micro-CT and AE in order to detect and analyse chloride-induced corrosion damage at the reinforcementconcrete interface. Second, a new signal-based clustering algorithm is developed in order to characterise different damage processes. Third, the developed algorithm was validated based on X-ray images and the occurence in time as well 
as the location of $\mathrm{AE}$ events. The results compared with alternative damage characterisation techniques based on AE parameters, that are found in the literature.

\section{Experimental Procedure}

\subsection{Materials and specimen preparation}

Cylindrical samples with a diameter of $36 \mathrm{~mm}$ and a height of $60 \mathrm{~mm}$ were made in a 3D printed mould. This sample size was required to obtain an appropriate resolution during micro-CT scanning. The sides of the mould were flattened to better attach the AE sensors afterwards. The mortar composition is given in table 1. Samples with and without reinforcement were made. In the ones with reinforcement, a smooth steel rod with a diameter of $6 \mathrm{~mm}$ was used. Both ends of the rebar were coated with an anti-corrosive paint. Only the middle part with a length of $20 \mathrm{~mm}$ was not coated. The bar was weighed before casting. Six mortar beams with no reinforcement were made in the same way to determine the flexural and compressive strength at 28 days and at the end of the corrosion test according to EN 12390-5 and EN 12390-3. All samples were stored in a curing room $\left(20 \pm 1^{\circ} \mathrm{C}, 95 \pm 3 \% \mathrm{RH}\right)$ for 28 days. At an age of 28 days, three beams were tested and the corrosion samples were completely immersed in a $5 \%$ sodium chloride solution for 24 hours. Accelerated corrosion and AE monitoring started on day 29. The experiment was repeated three times which means that three mortar batches were made. The mortar properties of every batch are given in table 2 .

Table 1: Mortar composition $\left[\mathrm{kg} / \mathrm{m}^{3}\right]$.

\begin{tabular}{llll}
\hline CEM I $42.5 \mathrm{~N}$ & Sand $(0 / 5)$ & Water & W/C \\
\hline 538 & 1344 & 269 & 0.5 \\
\hline
\end{tabular}

\subsection{Accelerated corrosion process}

The corrosion process was accelerated by an imposed direct current with a constant current density of $100 \mu \mathrm{A} / \mathrm{cm}^{2}$ as this is reported in the literature 
Table 2: Mortar properties at 28 days.

\begin{tabular}{|c|c|c|c|c|c|c|c|}
\hline \multirow[t]{2}{*}{ Material } & \multirow{2}{*}{$\begin{array}{l}\text { Amount } \\
\text { of tests }\end{array}$} & \multicolumn{2}{|c|}{ Batch $1\left[\mathrm{~N} / \mathrm{mm}^{2}\right]$} & \multicolumn{2}{|c|}{ Batch $2\left[\mathrm{~N} / \mathrm{mm}^{2}\right]$} & \multicolumn{2}{|c|}{ Batch $3\left[\mathrm{~N} / \mathrm{mm}^{2}\right.$} \\
\hline & & Mean & Std.Dev. & Mean & Std.Dev. & Mean & Std.Dev. \\
\hline $\begin{array}{c}\text { Compressive } \\
\text { strength }\end{array}$ & 6 & 43.96 & 2.99 & 40.40 & 0.43 & 46.40 & 1.49 \\
\hline $\begin{array}{l}\text { Flexural } \\
\text { strength }\end{array}$ & 3 & 7.09 & 0.17 & 7.60 & 0.54 & 6.78 & 0.83 \\
\hline
\end{tabular}

to be the maximum current density occurring in natural conditions [17]. For higher current densities, the internal pressure will be built up too quickly as the corrosion products will have no time to fill the pores [18. However, the formed corrosion products may be different from natural corrosion as the presence of oxygen may be limited. Different corrosion products will have different densities and therefore cause different expansion around the rebar. Determining the type of corrosion products was not performed during this investigation as the damage patterns are expected to be similar and will therefore not influence the results. The rebar was connected to the positive side of a DC regulator and acts as an anode. A stainless steel plate was connected to the negative side and acts as a cathode. During exposure, the specimen was partially immersed in a $5 \%$ sodium chloride solution. The setup is shown in figure 1 Each experiment had a duration of about one month in order to obtain an adequate corrosion level. The steel mass loss was estimated with Faraday's law (Eq. 1).

$$
\Delta m=\frac{t \cdot M \cdot I}{Z \cdot F},
$$

where $\Delta m$ is mass loss in [g], $t$ is time in [s], $M$ is molar mass of iron, $I$ is current in [A], $F$ is Faraday's constant which has the value $96487 \mathrm{C} / \mathrm{mol}$, and $Z$ is the valence. The valence describes how many electrons will be donated or received by the atom. In the case of iron, the valence can be 2 or 3 . In the corrosion cell, 2 electrons will be lost at the anodic site and gained at the cathodic site. Therefore, the valence will be 2 in this case. 


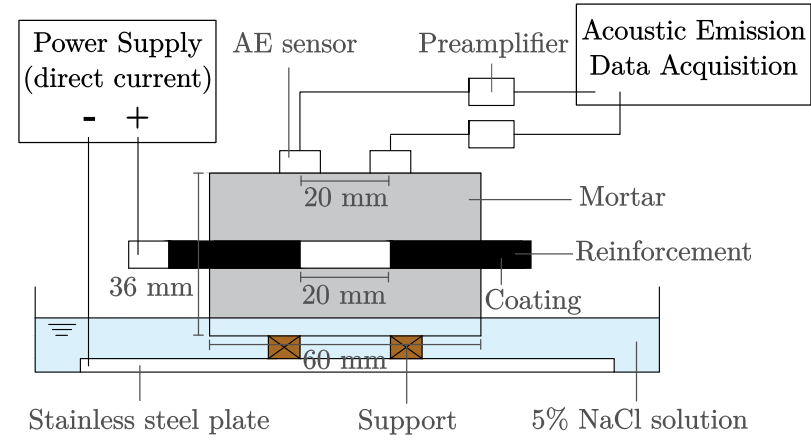

Figure 1: Accelerated corrosion setup and 1D AE sensor configuration.

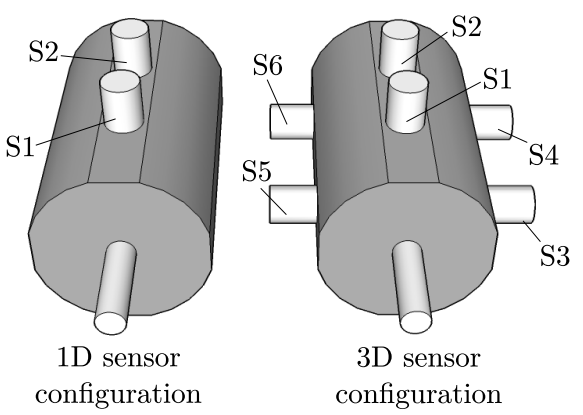

Figure 2: AE sensor (S) configurations. 


\subsection{Acoustic emission (AE) testing}

During the accelerated corrosion tests, the AE technique was used to monitor the specimens continuously. Piezoelectric broadband sensors having an operat-

90 glue and tape. Their wide frequency range allows a more reliable acquisition of different sources close to their original frequency. The sensors were connected to preamplifiers with $34 \mathrm{~dB}$ gain. These preamplifiers were connected to a Vallen AMSY-6 acquisition system with six AE channels. As the sample size is reof reflections. Therefore, the frequency spectrum of the acquisition system was set between $230 \mathrm{kHz}$ and $850 \mathrm{kHz}$. AE parameters and waveforms were then stored on a PC and the Vallen VisualAE software was used to process the data in real time. Matlab was used for further processing. The threshold was set to before the start of the test. For batch 1 and 3, three samples were monitored simultaneously by placing two sensors on top with a center-to-center distance of $20 \mathrm{~mm}$. One sample was corroding while the other ones were dummy samples. The purpose of the dummy samples was to investigate whether processes such as environmental noise, liquid absorption of the sodium chloride solution, or cement hydration are registered. For batch 2, one sample was monitored using six sensors. The corroding samples are indicated as B1-C1, B2-C1 and B3-C1 for batch 1, 2 and 3 respectively. An overview of the samples is given in table 3. The AE sensor configuration in $1 \mathrm{D}$ and $3 \mathrm{D}$ is shown in figure 2

\subsection{Microfocus X-ray Computed Tomography (micro-CT)}

$\mathrm{X}$-ray images were made at intermediate stages during the accelerated corrosion process. Before scanning, the AE sensors were removed, the sample was taken out of the solution and put on the rotating stage of the scanning machine. A Phoenix Nanotom system was used for data acquisition. As the image resolution is related to the specimen size, test scans were performed to optimise the setup and sample size and obtain adequate resolution for observing 
Table 3: Sample specifications.

\begin{tabular}{ccccc}
\hline Sample name & Batch & Rebar & Corroding/Dummy & Number of AE sensors \\
\hline B1-C1 & 1 & yes & corroding & 2 \\
B1-D1 & 1 & no & dummy & 2 \\
B1-D2 & 1 & no & dummy & 2 \\
B2-C1 & 2 & yes & corroding & 6 \\
B3-C1 & 3 & yes & corroding & 2 \\
B3-D1 & 3 & yes & dummy & 2 \\
B3-D2 & 3 & yes & dummy & 2 \\
\hline
\end{tabular}

the corrosion products and micro-cracking from an early stage [19. Since there is a large density difference between steel and mortar, a $0.3 \mathrm{~mm} \mathrm{Cu}$ filter was used in order to reduce artefacts such as beam hardening and streak artefacts. During each scan, 2400 images were taken over a rotation of $360^{\circ}$. The raw data consist of $2 \mathrm{D}$ projection images, which are used to reconstruct the object as a $3 \mathrm{D}$ array of density values. Afterwards, $2 \mathrm{D}$ slices can be made that can be analysed on the basis of different density values (difference in grey scale) in order to distinguish pores, cracks, corrosion products, mortar and steel.

Only the corroding samples were scanned. A single scan took about 2 hours. The applied voltage and current were respectively $180 \mathrm{kV}$ and $140 \mu \mathrm{A}$. The achieved resolution was $18 \mu \mathrm{m}$. Only the central portion of the sample with a height of $40 \mathrm{~mm}$ was scanned. Table 4 shows an overview of the performed scans, exposure time and total time since the start of the test. This was kept the same for every batch. Exposure time is defined as the time that the sample was corroding and AE monitoring was being performed. Total time is defined as the time since the start of the corrosion test and also includes the time needed for the micro-CT scans.

\subsection{Determination of corrosion level by gravimetric method}

135 After one month, the accelerated corrosion process was stopped and the rebars were removed from the samples by placing them in a $800^{\circ} \mathrm{C}$ oven for 
Table 4: Overview of scans, exposure time [hours] and total time [hours].

\begin{tabular}{ccc|ccc}
\hline Scan & Exposure time & Total time & Scan & Exposure time & Total time \\
\hline 1 & 0 & 0 & 5 & 395 & 410 \\
2 & 166 & 169 & 6 & 488 & 507 \\
3 & 234 & 241 & 7 & 556 & 579 \\
4 & 327 & 338 & 8 & 649 & 676 \\
\hline
\end{tabular}

two hours and subsequently breaking the mortar. The rebars were cleaned according to ASTM G1-76. Therefore, the rebars were put in Clark's solution for 25 minutes and weighed afterwards in order to determine the weight loss.

140 This process was repeated until no corrosion products could be distinguished under the microscope.

\section{AE data post-processing}

\subsection{Filtering}

The signals captured in the dummy samples were used to develop a filtering algorithm in Matlab to remove electrical noise and mechanical noise from processes in the lab environment. Examples of noisy signals are shown in figure 3. The signal was divided in two time windows. If the ratio between the amplitude of the first window and second window was smaller than 2 , the signal was seen as a noisy signal. Around $25 \%$ of the events were indicated as noisy signals. A similar approach based on the root mean square (RMS) of the signal was used by Benavent-Climent et al. [20].

\subsection{Arrival Time Picking}

One of the important factors that can affect the localisation accuracy of AE sources is the determination of the time of arrival (TOA) of the wave. Traditionally, the TOA is defined by the first threshold crossing which is sensitive to errors when it is not set to an optimal value. In this paper, the TOA was determined using the Akaike Information Criterion (AIC) 21] 22], as expressed 

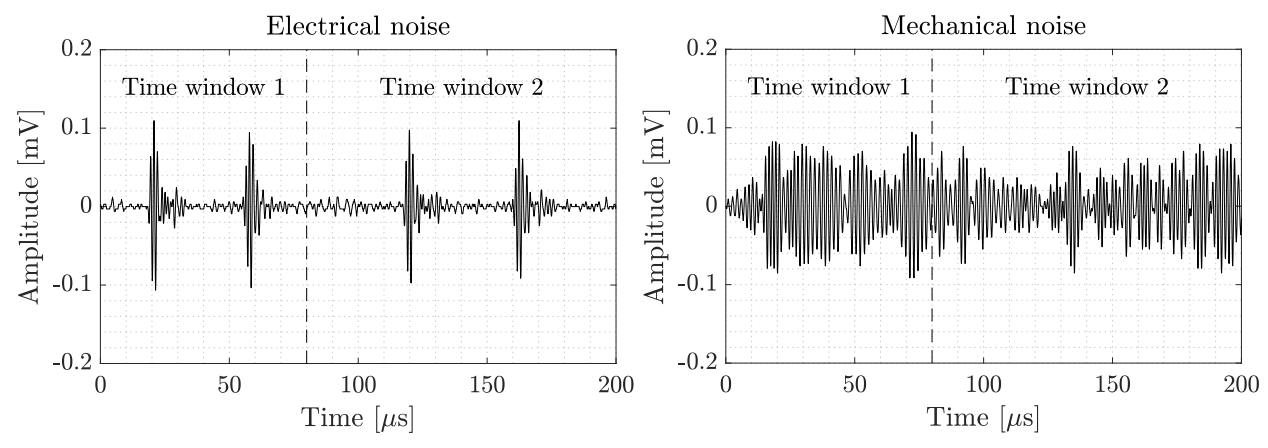

Figure 3: Example of electrical and mechanical noise signal.

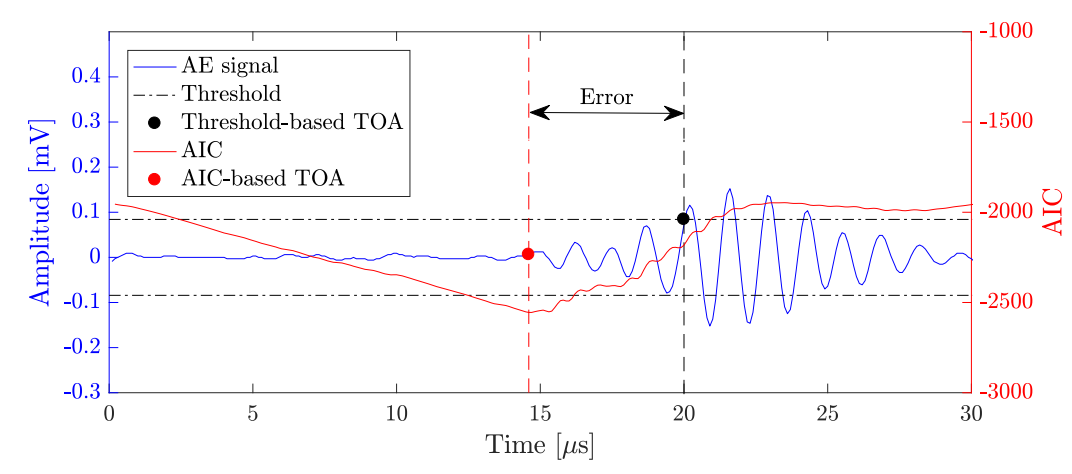

Figure 4: Difference between threshold-based arrival time picker and AIC picker.

in equation 2

$\operatorname{AIC}\left(t_{w}\right)=t_{w} \cdot \log _{10}\left(\operatorname{var}\left(R_{w}\left(1, t_{w}\right)\right)\right)+\left(T_{w}-t_{w}-1\right) \cdot \log _{10}\left(\operatorname{var}\left(R_{w}\left(t_{w}+1, T_{w}\right)\right)\right)$

The signal $R_{w}$ is divided into two sections at a point $t_{w}$. Point $t_{w}$ ranges from 1 to $T_{w}$, with $T_{w}$ the non-dimensional length of the time window. The term $\operatorname{var}\left(R_{w}\left(1, t_{w}\right)\right)$ is the variance function of all samples from 1 to $t_{w}$ and $\operatorname{var}\left(R_{w}\left(t_{w}+1, T_{w}\right)\right)$ is the variance function of all samples from point $t_{w}+1$ to $T_{w}$. The absolute minimum of all values indicates the onset time. The difference between a threshold-based arrival time picker and the AIC picker is exemplified in figure 4 


\subsection{Localisation of $A E$ events}

When using sensor 1 and sensor 2 (figure 2 of the samples, AE events can be localised in 1D. Therefore, three equations are used:

$$
\begin{gathered}
x_{1}=v \cdot\left(t_{1}-t_{0}\right) \\
x_{2}=v \cdot\left(t_{2}-t_{0}\right) \\
s=x_{1}+x_{2},
\end{gathered}
$$

where $s$ is the distance between the two sensors, $t_{1}$ and $t_{2}$ are the TOA at respectively sensor 1 and $2, t_{0}$ is the source time, and $v$ is the wave velocity. To solve these equations, only two sensors, placed on one line, are needed to localise $\mathrm{AE}$ events in 1D.

As 6 sensors are used on sample B2-C1, also localisation in 3D is possible for this sample. Therefore, an iterative approach, namely Geiger's method [23], was used. Geiger's method is a Gauss-Newton method for solving nonlinear problems.

The arrival time function for a homogeneous velocity model is as follows:

$$
f_{i}(x)=f_{i}(x, y, z, t)=t+\frac{1}{v_{i}} \sqrt{\left(x_{i}-x\right)^{2}+\left(y_{i}-y\right)^{2}+\left(z_{i}-z\right)^{2}},
$$

with $x, y$ and $z$ are the coordinates of an AE event, $t$ is the onset time of this event, $x_{\mathrm{i}}, y_{\mathrm{i}}$ and $z_{\mathrm{i}}$ are the coordinates of the $i^{\text {th }}$ sensor, and $v_{\mathrm{i}}$ is the wave velocity. Having four unknowns, information of at least four sensors is needed to solve the equations. Adding more sensors will enhance the localisation accuracy. A trial hypocenter $\left(x_{0}, y_{0}, z_{0}, t_{0}\right)$ in the middle of the specimen is chosen as a first guess of the source location. A correction factor is then calculated to minimise the travel time residuals which means that the calculated arrival times will best match the observed arrival times. Therefore, the function $f_{\mathrm{i}}(x)$ is expanded at a nearby location $x_{0}$. This expansion can be expressed by the 
first-degree Taylor polynomial:

$$
f_{i}(\mathbf{x})=f_{i}\left(\mathbf{x}_{\mathbf{0}}+\boldsymbol{\delta} \mathbf{x}\right)=f_{i}\left(\mathbf{x}_{\mathbf{0}}\right)+\frac{\partial f_{i}}{\partial x} \delta x+\frac{\partial f_{i}}{\partial y} \delta y+\frac{\partial f_{i}}{\partial z} \delta z+\frac{\partial f_{i}}{\partial t} \delta t=f_{i}\left(\mathbf{x}_{\mathbf{0}}\right)+\gamma_{i}
$$

or in matrix notation:

$$
\mathbf{A} \delta \mathbf{x}=\gamma
$$

where $A$ is the matrix of partial derivatives, $\delta \mathrm{x}$ the correction vector and $\gamma$ the residual vector.

The correction vector is then calculated by the Moore-Penrose inverse to compute the least squares solution:

$$
\delta \mathbf{x}=\left(\mathbf{A}^{\mathbf{T}} \mathbf{A}\right)^{-1} \mathbf{A}^{\mathbf{T}} \gamma
$$

The trial solution is updated at the beginning of each iteration step by adding the correction factor from the previous iteration.

Two criteria were set to stop the iterative process. The first stopping criterion is based on the size of the correction vector. The correction vector $\boldsymbol{\delta} \mathbf{x}$ include both spatial and time components which makes it difficult to calculate its size as it contains different units 23 . Therefore, the correction vector $\boldsymbol{\delta} \mathbf{d}$ was determined only based on the spatial components:

$$
\delta \mathbf{d}=\sqrt{\delta x^{2}+\delta y^{2}+\delta z^{2}}
$$

The correction vector should be smaller than a chosen tolerance $\epsilon$. When $\boldsymbol{\delta} \mathbf{d}$ goes to zero, it is a sign of convergency. Therefore the tolerance $\epsilon$ was chosen to be 0.02 . The maximum amount of iterations was set to 10 .

\subsection{Clustering}

In literature, $\mathrm{AE}$ analysis is mainly performed based on $\mathrm{AE}$ parameters which allows to distinguish different stages of the reinforcement corrosion such as the initiation (before cracking) and propagation (after cracking) stage [8]. Signal-based analysis allows to distinguish different damage processes such as the corrosion process itself and mortar cracking leading to a more reliable interpretation of the different damage mechanisms. A novel dedicated signal-based 
clustering algorithm is developed by the authors to characterise the different $\mathrm{AE}$ signals during the accelerated corrosion tests. Clustering is the process in which a set of objects or points are grouped into categories or clusters according to a virtual distance measure. Similar points in the same cluster will have a small distance to each other while points in different clusters are dissimilar and thus are at a large distance from one another [24].

In signal-processing, cross-correlation can be used as a measure of similarity of two waveforms as a function of a time-lag applied to one of them. It is also known as the sliding dot product or sliding inner-product. For continuous functions $f(t)$ and $g(t)$, the cross-correlation is defined as:

$$
R_{f g}(t)=(f \star g)(t)=\int_{-\infty}^{+\infty} f(\tau) g(t+\tau) d \tau
$$

Function $g(t)$ is slided along the $x$-axis, calculating the integral of their product at each position. The value of $(f \star g)(t)$ is maximised when the functions match. The contribution to the integral is large when peaks (positive areas) or troughs (negative areas) align. Referring to seismology, normalised crosscorrelation is frequently used because two time series having a high amplitude will give large correlation values. Therefore, the similarity cannot be determined by comparing the amplitude of the cross-correlation. The normalised crosscorrelation $\hat{R}_{f g}$ is given in Eq. 12 and will vary between -1 and 1 . The value 1 means that the two signals have the same shape, -1 means that they have the same shape but opposite signs and 0 means that they are not similar at all.

$$
\hat{R}_{f g}(t)=\frac{R_{f g}(t)}{\sqrt{R_{f f}(0) R_{g g}(0)}}
$$

where

$$
\begin{aligned}
& R_{f f}(0)=\int_{-\infty}^{+\infty} f(\tau)^{2} d \tau \\
& R_{g g}(0)=\int_{-\infty}^{+\infty} g(\tau)^{2} d \tau
\end{aligned}
$$

Afterwards, the normalised cross-correlation coefficient $c c$ can be calculated as follows:

$$
c c(f, g)=\max \left(\hat{R}_{f g}(t)\right)
$$




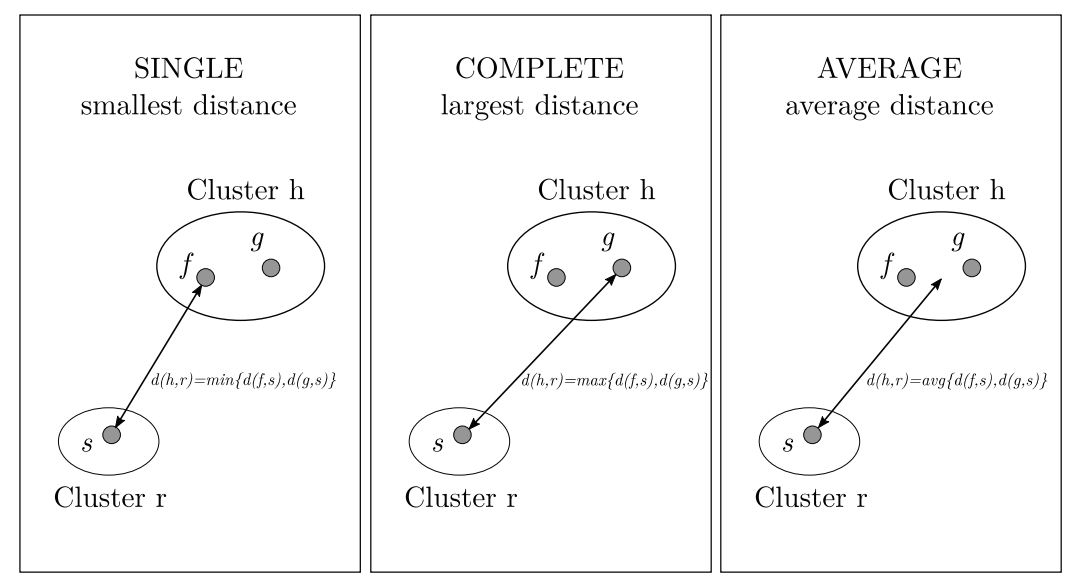

Figure 5: Different linkage methods for hierarchical clustering.

In clustering analysis, dissimilarity can be used as a distance measure. The distance or dissimilarity $d(f, g)$ can be defined as 1 minus the correlation coefficient (Eq. 16). According to this definition, the more similar two signals are, the shorter their distance will be. The distance approaches 0 as correlation goes to 1 .

$$
d(f, g)=1-c c(f, g)
$$

In this paper, a signal-based hierarchical clustering algorithm was developed and implemented. Each signal forms a separate cluster at the start. These onesignal-clusters are then combined into larger clusters based on their closeness. Two clusters with the shortest distance, i.e. smallest dissimilarity, are merged first. Then, the distance between this newly formed cluster and the other existing clusters needs to be calculated. The calculation of this inter-cluster distance or cophenetic distance can be executed in different ways, among which single (nearest neighbour), complete (furthest neighbour), and average linkage are most commonly used (figure 5) 24]. Average linkage is used in this paper as single linkage can produce chain-shape clusters and complete linkage is more sensitive to outliers.

Afterwards, the two clusters with the smallest distance are combined and again the cophenetic distance is calculated. These steps are repeated until only 


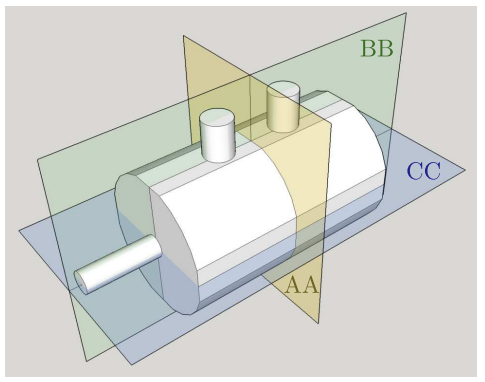

Figure 6: Indication of different slices made through the samples.

one cluster is formed after $1-N$ iterations with $N$ the amount of signals. The result can be visualised in a tree structure plot called dendrogram.

\section{Results and discussion}

205 ages. For the analysis of the micro-CT results, different slices are made through the sample using the software package Dataviewer. The different directions are shown in figure 6. Slices AA are made perpendicular to the rebar, slices BB are parallel to the rebar and sensor position, and slices CC are parallel to the rebar ple B1-C1, B2-C1 and B3-C1. The result for B2-C1 was obtained by only taking into account the $\mathrm{AE}$ events from the sensors placed on top of the sample (sensor 1 and sensor 2). The AE energy is calculated by squaring and integrating the $\mathrm{AE}$ signals. The energy unit eu is equal to $10^{-14} \mathrm{~V}^{2}$ or $10^{-18} \mathrm{~J}$. The amount

of AE energy is larger for B2-C1 than for B1-C1 and B3-C1. The moment of cracking of B1-C1 and B2-C1 can clearly be distinguished by the sudden jump in AE energy. This is not the case for sample B3-C1 where the AE energy 


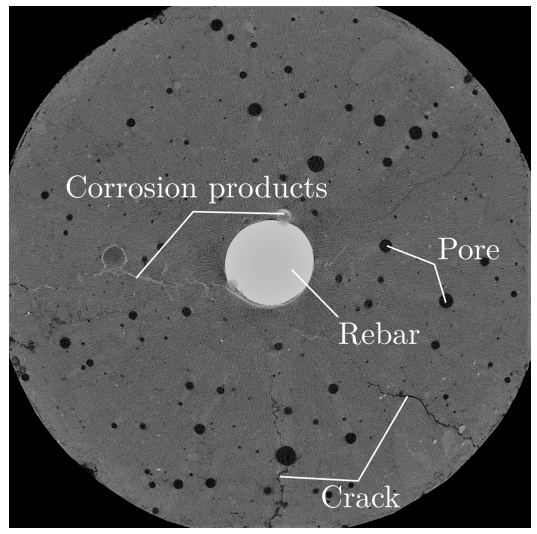

Figure 7: Cross section (AA) of sample B1-C1 showing different phases.

steadily increases. No cracks could be distinguished on the CT images of this sample. The corrosion levels were also confirmed by the mass loss, given in table 5. The mass loss was determined in three different ways: by estimation with Faraday's law, by weighing the cleaned rebar and by volume calculation from the CT images. A difference can be noticed between the different measurement techniques. Faraday's law assumes that all current is applied to dissolve iron in the electrochemical reaction which is not the case in reality. Parameters that influence the corrosion onset in reinforced concrete such as concrete cover thickness and porosity, are also not taken into account in this equation. For the weighing (gravimetric method), the entire rebar was cleaned. Also some metal of the non-corroding zones will dissolve. Therefore, the highest mass loss values were obtained with this technique. For micro-CT, the mass loss is underestimated, probably due to segmentation errors. Due to the high density of steel, corrosion products appear to have the same density (shielding effect). Part of the corrosion products was also seen as steel during segmentation. Also the resolution of the images can cause this difference. Overall, the different measurement techniques show a similar trend, being that the mass loss of B1-C1 and $\mathrm{B} 2-\mathrm{C} 1$ is higher than for $\mathrm{B} 3-\mathrm{C} 1$ which confirms the $\mathrm{AE}$ results.

The cumulative AE energy versus time for B1-C1 is given in figure 9 (top). The results are shown against the mass loss of the rebar which was calculated 


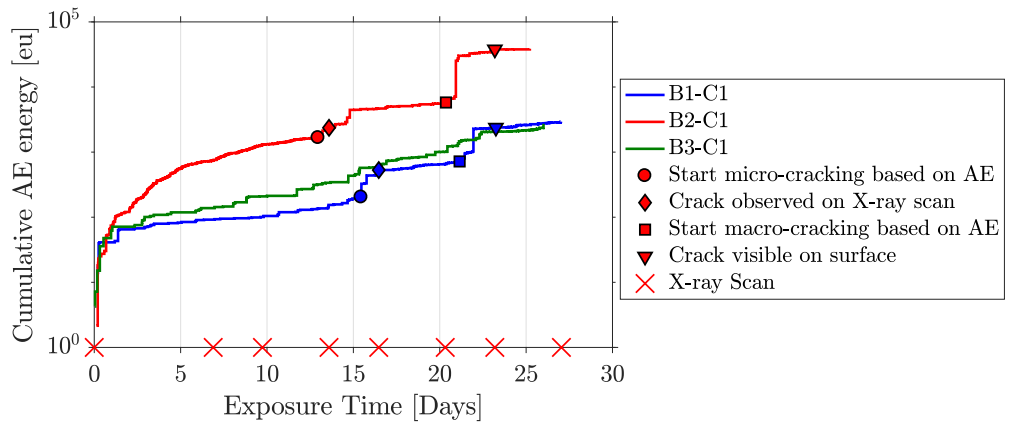

Figure 8: Time history of cumulated AE energy of B1-C1, B2-C1 and B3-C1 with indication of moment of micro-crack initiation and macro-crack initiation as observed from micro-CT images and visual inspection.

Table 5: Comparison of mass loss (g) obtained by Faraday's law, by weighing and micro-CT analysis.

\begin{tabular}{c|ccc}
\hline & B1-C1 & B2-C1 & B3-C1 \\
\hline Faraday's law & 0.26 & 0.26 & 0.26 \\
Weighing & 0.34 & 0.43 & 0.16 \\
Micro-CT & 0.12 & 0.12 & 0.02 \\
\hline
\end{tabular}

from the micro-CT imaging using software package $C T A n$. A $3 \mathrm{D}$ view obtained from micro-CT imaging of the rebar is also shown at the bottom of the figure.

245 A good agreement between the AE energy and mass loss is noticeable in terms of slope increase of the AE energy curve. Micro-crack initiation in the mortar can clearly be distinguished from the AE data by an increase in AE energy and is indicated by the light grey area. Macro-cracking is indicated by the dark grey area. A similar trend was observed when looking at the amount of events. These observations are also confirmed by the CT scans, shown in the middle of figure 9. Before cracking, the $\mathrm{AE}$ events are mainly induced by the corrosion process and internal pressure caused by the corrosion products.

For B1-C1, AE sources were located using the linear location algorithm described in 3.3 as two sensors were placed on the sample surface. The localised events were correlated with micro-CT images at different points in time. 


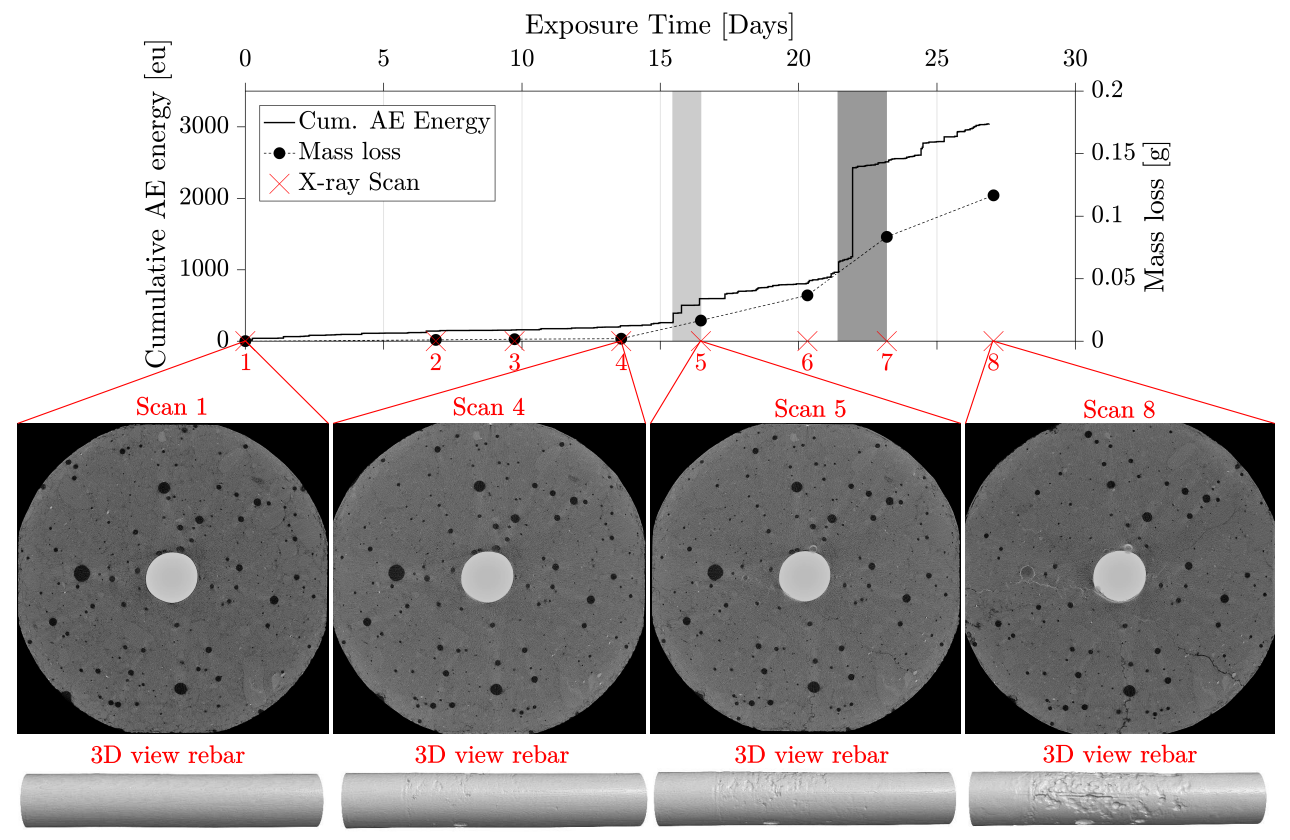

Figure 9: Top: Time history of cumulative AE energy of B1-C1; Middle: Sections (AA) at different time intervals; Bottom: 3D view of the rebar at different time intervals. 

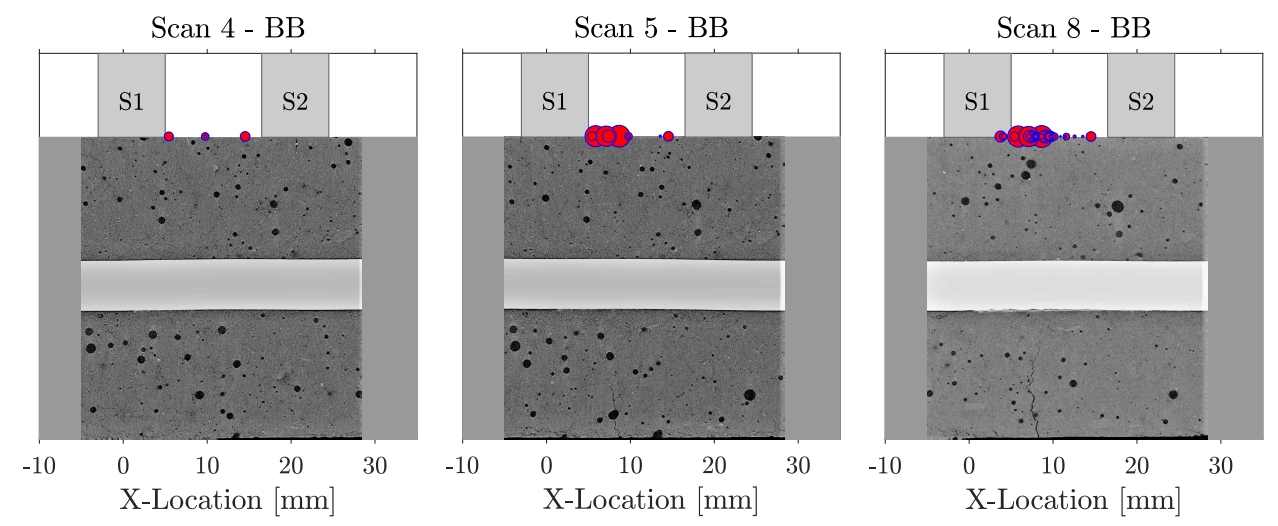

AE energy $[\mathrm{eu}]$

Figure 10: Lateral section (BB) along the rebar of B1-C1 with indication of localised AE events in 1D (along $\mathrm{X}$-axis).

Figure 10 shows a lateral cross section along the rebar (direction BB) during three scanning moments: scan 4 (before micro-cracking), scan 5 (after microcracking), and scan 8 (at the end of the test). The sensors are indicated in grey on top of the sample. The $1 \mathrm{D}$ location of the AE events along the $\mathrm{X}$-axis is represented by red dots. The size of the dots depends on the amount of $\mathrm{AE}$ energy: the diameter increases as the amount of $\mathrm{AE}$ energy is larger. It can be noticed that only few, low-energy events are localised before cracking (scan 4). The location is in good agreement with the observed damage seen on the CT scans. The AE sensors that are connected on top of the sample are able to locate the damage at the bottom side of the rebar. This is very promising for the future on-site use of the technique when for example a structural component is only reachable from one side.

The clustering algorithm is investigated for sample B2-C1. The clustering algorithm was applied on the localised events. The first hit of the 3D localised events was used to calculate the correlation coefficients. The AE signal $V(t)$ is a convolution of the input function or source $S(t)$, the structure function $G(t)$ which depends on the travel path of the wave and thus the geometry and material of the sample, and the recording system factor $R(t)$ which takes into 
account the frequency responses of the couplant, the sensor, recording system and amplifier (Eq. 17). When using the first hit of each event instead of the hit registered by the same channel, the signal is less affected by the travel path of the wave as the sensor closest to the source is chosen.

$$
V(t)=S(t) * G(t) * R(t)
$$

From figure 11, two clusters can be separated. The threshold to distinguish the clusters was set to a dissimilarity of 0.70 (black dashed line). The

(FFT). Higher frequencies and lower RA-values are typically assigned to the tensile mode whereas lower frequencies and higher RA-values to the shear mode. 


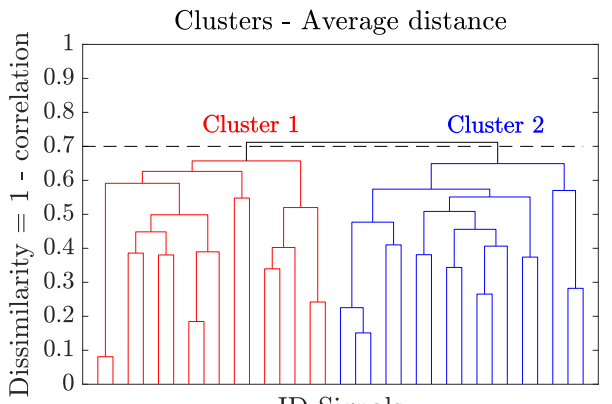

ID Signals

Figure 11: Dendrogram showing two different clusters.

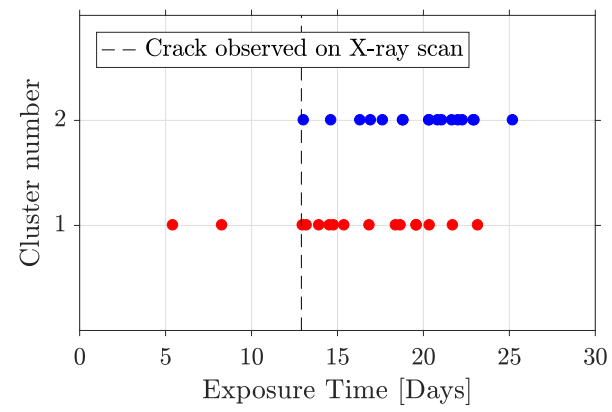

Figure 12: Events of different clusters versus time with indication of the moment of cracking as observed on the micro-CT images.

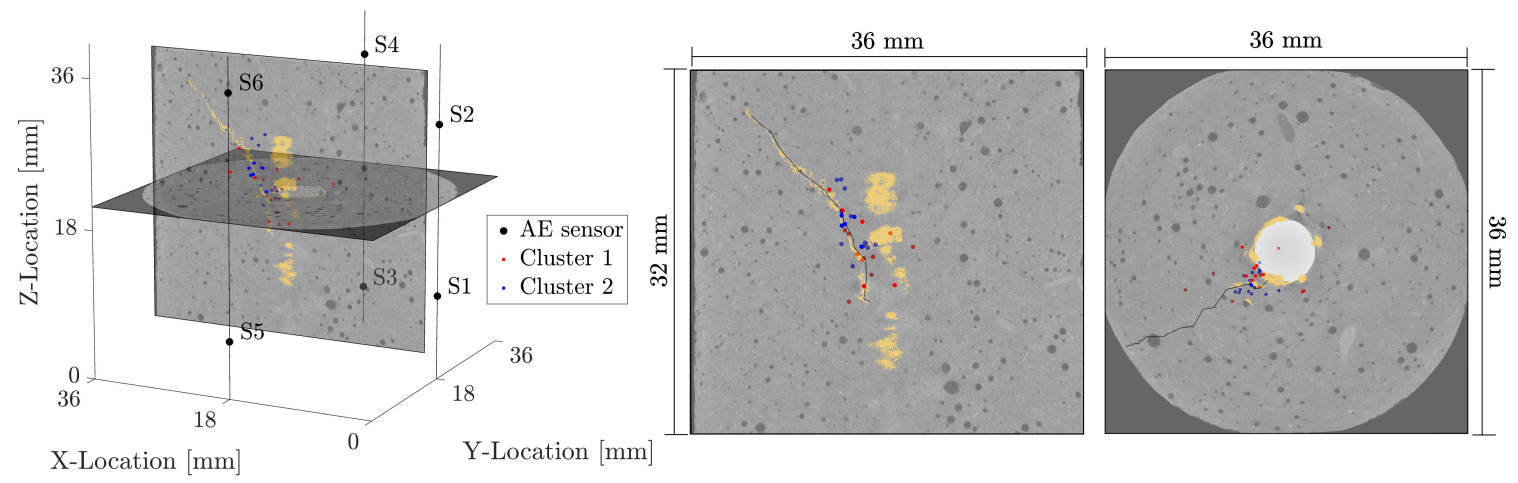

Figure 13: Left: 3D view of localised events and micro-CT images; middle: view of localised events and crack (black line) on slice in direction BB; right: view of localised events around rebar on slice in direction AA. 

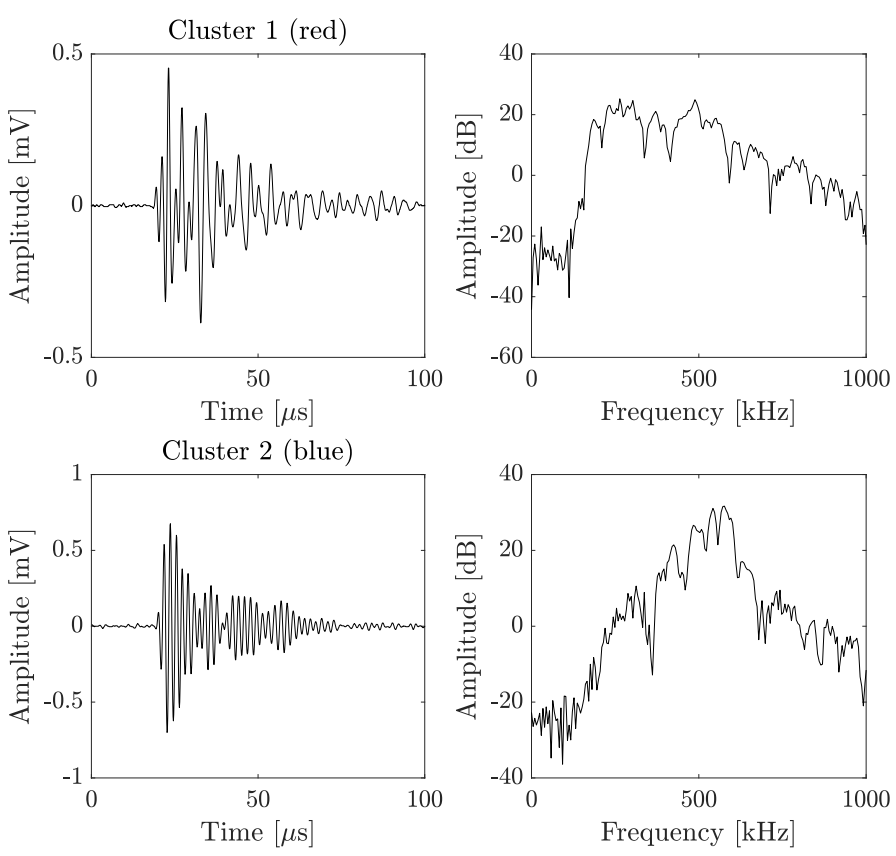

Figure 14: Typical signals of the different clusters.

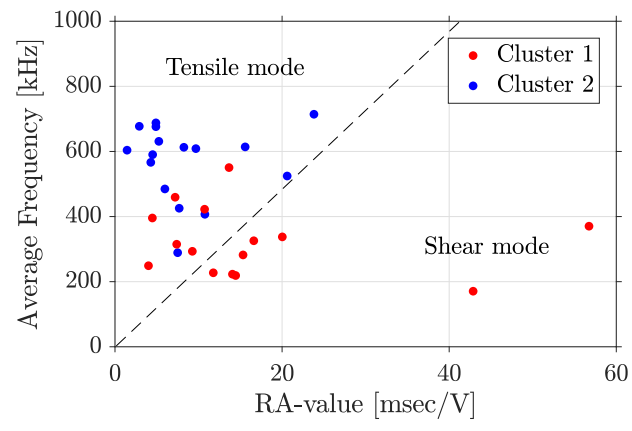

Figure 15: Relationship between RA-value and peak frequency of clustered events. 
It was found in the literature that the shear mode can mainly be distinguished during the onset of corrosion whereas the tensile mode is generated during cracking [28. Also in this paper, seen on figure 15, it is suggested that the tensile mode can be assigned to cluster 2 (blue) and the shear mode to most of the signals of cluster 1 (red).

\section{Conclusions}

In this paper, the Acoustic Emission (AE) technique was used for corrosion monitoring and was validated with images obtained with X-ray computed tomography (micro-CT). Following conclusions can be drawn:

1.) The comparison between different samples shows that AE monitoring during the corrosion process is able to quantify the amount of corrosion damage. The moment of cracking can clearly be distinguished from the change in AE energy allowing to differentiate between cracked and uncracked samples.

2.) The localised $\mathrm{AE}$ events in $1 \mathrm{D}$ and $3 \mathrm{D}$ accord to the damage observed on the CT images. Thus, AE allows to accurately localise damage due to corrosion in reinforced concrete.

3.) The implemented clustering algorithm was able to distinguish two different clusters, representing different types of AE signals. Based on the occurrence in time and location, possible damage processes were assigned allowing to reliably interpret the different ongoing processes.

Results during continuous monitoring and on small-scale samples are very promising. Further research will focus on the up-scaling of the results. Also different monitoring schemes will be investigated.

\section{Acknowledgments}

This research was performed within the framework of project C24/17/042 "Multi-scale assessment of residual structural capacity of deteriorating reinforced concrete structures", supported by Internal Funds KU Leuven. 


\section{References}

[1] H. Song, V. Saraswathy, Corrosion monitoring of reinforced concrete structures - a review., International Journal of Electrochemical Science 2 (2007) $1-28$.

[2] A. Zaki, H. K. Chai, D. G. Aggelis, N. Alver, Non-destructive evaluation for corrosion monitoring in concrete: A review and capability of acoustic emission technique., Sensors 15(8) (2015) 19069-19101.

[3] M. Wevers, Listening to the sound of materials: Acoustic emission for the analysis of material behaviour., NDT and E International 30(2) (1997) 99106.

[4] C. Jirarungsatian, A. Prateepasen, Pitting and uniform corrosion source recognition using acoustic emission parameters., Corrosion Science 52 (2010) 187-197.

[5] R. K. Miller, A. A. Pollock, D. J. Watts, J. M. Carlyle, A. N. Tafuri, J. Yezzi Jr, A reference standard for the development of acoustic emission pipeline leak detection techniques., NDT and E International 32(1) (1999) $1-8$.

[6] D. J. Yoon, W. J. Weiss, S. P. Shah, Assessing damage in corroded reinforced concrete using acoustic emission., ASCE Journal of Engineering Mechanics 126(3) (2000) 273-283.

${ }_{340}$ [7] Y. Kawasaki, T. Wakadu, T. Kobarai, M. Ohtsu, Corrosion mechanisms in reinforced concrete by acoustic emission., Construction and Building Materials 48 (2013) 1240-1247.

[8] H. Idrissi, A. Limam, Study and characterization by acoustic emission and electrochemical measurements of concrete deterioration caused by reinforcement steel corrosion., NDT and E International 36 (2003) 563-569. 
[9] M. Wevers, P. de Meester, R. Swennen, Microfocus x-ray computed tomography in materials research., Insight 43(10) (2001) 658-663.

[10] V. Cnudde, J. Dewanckele, M. Boone, T. de Kock, M. Boone, L. Brabant, M. Dusar, M. De Ceukelaire, H. De Clercq, R. Hayen, P. Jacobs, High-resolution x-ray ct for $3 \mathrm{~d}$ petrography of ferruginous sandstone for an investigation of building stone decay., Microscopy Research and Technique 74(11) (2011) 1006-1017.

[11] E. Verstrynge, C. Van Steen, J. Andries, K. Van Balen, L. Vandewalle, M. Wevers, Experimental study of failure mechanisms in brittle construction materials by means of x-ray microfocus computed tomography., Proceedings of the 9th International Conference on Fracture Mechanics of Concrete and Concrete Structures, Berkeley, USA (2016) 1-11.

[12] A. Michel, P. B. J., M. R. Geiker, H. Stang, J. F. Olesen, Monitoring reinforcement corrosion and corrosion-indced cracking using non-destructive x-ray attenuation measurements., Cement and Concrete Research 41(11) (2011) 1085-1094.

[13] B. Savija, M. Lukovic, S. A. S. Hosseini, J. Pacheco, E. Schlangen, Corrosion induced cover cracking studied by x-ray computed tomography, nanoindentation, and energy dispersive x-ray spectrometry, Materials and Structures 48(7) (2015) 2043-2062.

[14] T. Suzuki, H. Ogata, R. Tadaka, M. Aoki, M. Ohtsu, Use of acoustic emission and x-ray computed tomography for damage evaluation of freezethawed concrete., Construction and Building Materials 24 (2010) 23472352.

[15] T. J. Chotard, A. Smith, M. P. Boncoeur, D. Fargeot, C. Gault, Characterisation of early stage calcium aluminate cement hydration by combination of non-destructive techniques: acoustic emission and x-ray tomography., Journal of the European Ceramic Society 23(13) (2003) 2211-2223. 
[16] E. Verstrynge, R. Adriaens, J. Elsen, K. Van Balen, Multi-scale analysis

[17] C. Andrade, C. Alonso, Corrosion rate monitoring in the laboratory and on-site., Construction and Building Materials 10(5) (1996) 315-328.

[18] S. Caré, A. Raharinaivo, Influence of impressed current on the initiation of on the influence of moisture on the mechanical behavior of ferruginous sandstone., Construction and Building Materials 54 (2014) 78-90. damage in reinforced mortar due to corrosion of embedded steel., Cement and Concrete Research 37 (2007) 1598-1612.

[19] C. Van Steen, M. Wevers, E. Verstrynge, X-ray computed tomography for the detection of corrosion-induced damage at the reinforcement-concrete interface., Proceedings of the 7th Conference on Industrial Computed Tomography, Leuven, Belgium (2017) 1-10.

[20] A. Benavent-Climent, A. Gallego, J. M. Vico, An acoustic emission energy index for damage evaluation of reinforced concrete slabs under seismic loads., Structural Health Monitoring 11 (2011) 69-81.

[21] H. Akaike, Markovian representation of stochastic processes and its application to the analysis of autoregressive moving average processes., Annals of the Institute of Statistical Mathematics 26(1) (1974) 363-387.

[22] J. H. Kurz, C. U. Grosse, H.-W. Reinhardt, Strategies for reliable automatic onset time picking of acoustic emissions and of ultrasound signals in concrete., Ultrasonics 43 (2005) 538-546.

[23] M. Ge, Analysis of source location algorithms: Part ii: Iterative methods., Journal of Acoustic Emission 21 (2003) 29-51.

[24] O. Maimon, L. Rokach, The Data Mining and Knowledge Discovery Handbook, Springer, New York, 2005.

[25] W. Li, C. Xu, S. C. M. Ho, B. Wang, G. Song, Monitoring concrete deterioration due to reinforcement corrosion by integrating acoustic emission and fbg strain measurements., Sensors 17 (2017) 1-12. 
[26] M. Di Benedetti, G. Loreto, Matta, A. Nanni, Acoustic emission historic index and frequency spectrum of reinforced concrete under accelerated corrosion., Journal of Materials in Civil Engineering 26(9) (2014) 1-8.

[27] Z. Li, F. Li, A. Zdunek, E. Landis, S. P. Shah, Application of acoustic emission technique to detection of reinforcing steel corrosion in concrete., ACI Materials Journal 95 (1998) 68-81.

[28] V. Leelalerkiet, T. Shimizu, Y. Tomoda, M. Ohtsu, Estimation of corrosion in reinforced concrete by electrochemical techniques and acoustic emission., Journal of Advanced Concrete Technology 3(1) (2005) 137-147. 